\title{
Limbic System Associated Membrane Protein Mutation in an Iranian Family Diagnosed with Ménière's Disease
}

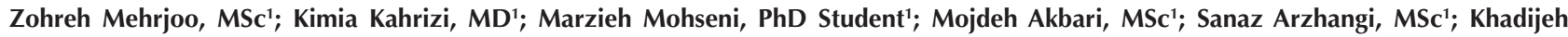 \\ Jalalvand'; Hossein Najmabadi, PhD'; Mohammad Farhadi, MD²; Mohammad Mohseni, MD²; Alimohamad Asghari, MD³; Saleh Mohebbi, \\ $\mathrm{MD}^{3}$; Ahmad Daneshi, $\mathrm{MD}^{2 *}$
}

${ }^{1}$ Genetics Research Center, University of Social Welfare and Rehabilitation Sciences, Tehran, Iran

${ }^{2}$ ENT and Head \& Neck Research Center and Department, Hazrat Rasoul Hospital, The Five Senses Institute, Iran University of Medical Sciences, Tehran, Iran

${ }^{3}$ Skull Base Research Center, The Five Senses Institute, Iran University of Medical Sciences, Tehran, Iran

\begin{abstract}
Background: Ménière's disease (MD) is a common inner ear disorder which is characterized by recurrent attacks of vertigo, fluctuating sensorineural hearing loss (SNHL), tinnitus, and a sense of fullness in the affected ear. MD is a complex disorder; although six genes have been linked to familial autosomal dominant form of the disease, in many cases, the exact genetic etiology remains elusive.

Methods: To elucidate the genetic causes of MD in an Iranian family, we performed exome sequencing on all members of the family: consanguineous parents and four children (two affected and two unaffected). Variant filtering was completed using a customized workflow keeping variants based on segregation with MD in autosomal recessive (AR) inheritance pattern, minor allele frequency (MAF), and in-silico prediction of pathogenicity.

Results: Analysis revealed that in this family, 970 variants co-segregated with MD in AR pattern, out of which eight variants (one intergenic, four intronic, and three exonic) were extremely rare. The exonic variants included a synonymous substitution in USP3 gene, an in-frame deletion in ZBED2 gene, and a rare, highly conserved deleterious missense alteration in LSAMP gene.

Conclusion: The phenotype observed in the proband described here, i.e. vertigo, poor sense of smell, tinnitus, and borderline hearing ability, may originate from aberrant changes in the cerebellum and limbic system due to a deleterious mutation in the LSAMP gene; hence, LSAMP mutation is a possible candidate for the etiology of MD in this family.

Keywords: Autosomal Recessive, Exome sequencing, Familial Ménière's disease, Genetics

Cite this article as: Mehrjoo Z, Kahrizi K, Mohseni M, Akbari M, Arzhangi S, Jalalvand K, et al. Limbic system associated membrane protein mutation in an Iranian family diagnosed with Ménière's disease. Arch Iran Med. 2020;23(5):319-325. doi: 10.34172/ aim.2020.21.
\end{abstract}

Received: July 1, 2019, Accepted: January 26, 2020, ePublished: May 1, 2020

\section{Introduction}

Diseases which display phenotypic heterogeneity, pleiotropy and incomplete penetrance, often arise from a complex combination of etiological factors, including genetic and environmental components. As such, these diseases are often hard to characterize and consequently, it is difficult to elucidate the genetics and pathophysiology underlying the disease, as is the case with Ménière's disease (MD [MIM 156000]). MD is a complex disease which displays considerable clinical heterogeneity. The broad clinical spectrum of symptoms includes episodes of spontaneous vertigo lasting from 20 minutes to several hours ${ }^{1,2}$ becoming less frequent but more severe over time. $^{3}$ Sensorineural hearing loss (SNHL) typically begins at low frequencies and fluctuates, ultimately resulting in progressive moderate to severe hearing loss. ${ }^{1,3-5}$ Alongside vertigo and SNHL, patients often experience aural fullness and tinnitus, an imaginary but debilitating sensation of ringing, buzzing, or hissing. MD symptoms mainly affect one ear (unilateral MD); however, bilateral MD has also been reported. ${ }^{1,5,6}$ Broadly, MD falls into two clinical categories: definite and probable, with probable being less phenotypically severe. ${ }^{7} \mathrm{MD}$ usually begins to manifest in the third to the seventh decades of life with a small female predominance, and only a few rare cases have been identified in younger people. . $^{2,78}$

The prevalence of MD is estimated to be roughly $0.5-$ 1/1000 individuals. ${ }^{1,6,7,9,10}$ Among these, approximately $10 \%$ are familial MD (FMD), ${ }^{4}$ in which at least one other relative (first- or second-degree) fulfills all of the criteria for definite or probable MD., ${ }^{3,4,11}$ While FMD and sporadic MD exhibit the same phenotypes, there is often greater severity and earlier onset in FMD. ${ }^{3,4,8,12}$ FMD has been described in families originating from the UK, Brazil, Sweden, Finland, Germany, and Spain..$^{3,4,6,10-13}$ Outside the Caucasian population, MD is rare or virtually absent in 
many populations. ${ }^{2,12}$ It is considered to be a multifactorial disorder and the clinical heterogeneity observed among patients points to the suggested etiological diversity including environmental and genetic factors, dysfunction of the immune system, and viral infections. ${ }^{4,9}$ The fact that approximately one-third of MD patients have family members with MD-like symptoms, and different MD incidences in different populations lend credence to the role of genetics in the development of MD. ${ }^{11}$

MD can be inherited in autosomal dominant (AD), and rarely in mitochondrial or recessive patterns. ${ }^{3,4,6,12,13}$ Mutations in DTNA, FAM136A, COCH, DPT, $S E M A 3 D$, and $P R K C B$ genes have been described in ADFMD ${ }^{2,14-16}$; however, no gene has been identified for recessive inheritance. Here, we describe a consanguineous Iranian family with autosomal recessive (AR) FMD. After detailed genetic analysis, we conclude that a mutation of the gene encoding the limbic system associated membrane protein $(L S A M P)$ plays a role in ARFMD.

\section{Materials and Methods}

An Iranian Lur family (L-8600471) with apparent ARFMD was selected for this study. Clinical examination, including brain MRI, was performed. Pure tone audiometry was carried out to determine air conduction thresholds at 0.25 , $0.5,1,2,3,4$ and $8 \mathrm{kHz}$. After obtaining informed consent, whole blood samples were collected from all members of the core family including both individuals affected with ARFMD, unaffected siblings and both parents. DNA was extracted using the conventional salting out method. ${ }^{17}$ All procedures were approved by the human research institutional review boards at the University of Social Welfare and Rehabilitation Sciences and Iran University of Medical Sciences, Tehran, Iran.

Targeted genomic enrichment (TGE) was performed using either the Agilent SureSelect Human All Exon kit (v.5) or the Agilent SureSelect XT Human All Exon kit (v.6) (Agilent Technologies, Inc., Santa Clara, CA, USA). Captured genomic libraries underwent subsequent massively parallel sequencing (MPS) using the Illumina HiSeq2000 or the Illumina NextSeq 500 platform (Illumina Inc., San Diego, CA, USA). After sequencing, raw reads were aligned to the hg19 build of the human reference genome using Burrows-Wheeler Aligner (BWA), MEM algorithm. ${ }^{18}$ Data processing and variant discovery (SNPs and Indels) were performed according to the best practices workflow of the Genome Analysis Toolkit (GATK). ${ }^{19,20}$ Variants were listed in a gVCF file per sample which were then merged together. Variant annotation was performed using ANNOVAR software. ${ }^{21}$ Annotated variants underwent filtering and prioritization based on the AR mode of inheritance. Those variants were kept for which both parents were heterozygous, both healthy siblings were wild type homozygous or heterozygous, and both affected siblings were mutated homozygous. Variants were further prioritized considering their frequencies in the 1000 Genome project, ExAC, ESP6500, genomAD, exomAD, and Iranome databases (http://iranome. $\mathrm{com} /) .{ }^{22-24}$ The prioritizing cut-off started at minor allele frequency $(\mathrm{MAF})<0.05$ and then narrowed down to 0.01 , 0.001 , and finally 0.0001 .

\section{Results}

Family L-8600471 originates from western Iran and is of Lur ethnicity with consanguineous parents (Figure 1a). Brain MRI was normal in proband IV:3. Audiological examination revealed borderline hearing in both ears of proband IV:3 (Figure 1b). She complained of vertigo, a sense of vomiting, tinnitus, hearing impairment, and poor sense of smell, with onset around 30 years of age. After clinical examination, she was diagnosed with definite MD. The same phenotypes, apart from a poor sense of smell, were reported in the proband's sister (IV:4) with onset at 45 years of age; she also had a history of depression.

After applying variant filtering, as described in methods, 970 variants remained that were inherited in an AR pattern. Then, the variants were prioritized in terms of MAF based on publicly available databases of the 1000 Genome project, ESP6500, ExAC, gnomAD genome, and gnomAD exome $(<5 \%,<1 \%,<0.1 \%$, and $<0.01 \%)$,

(a)
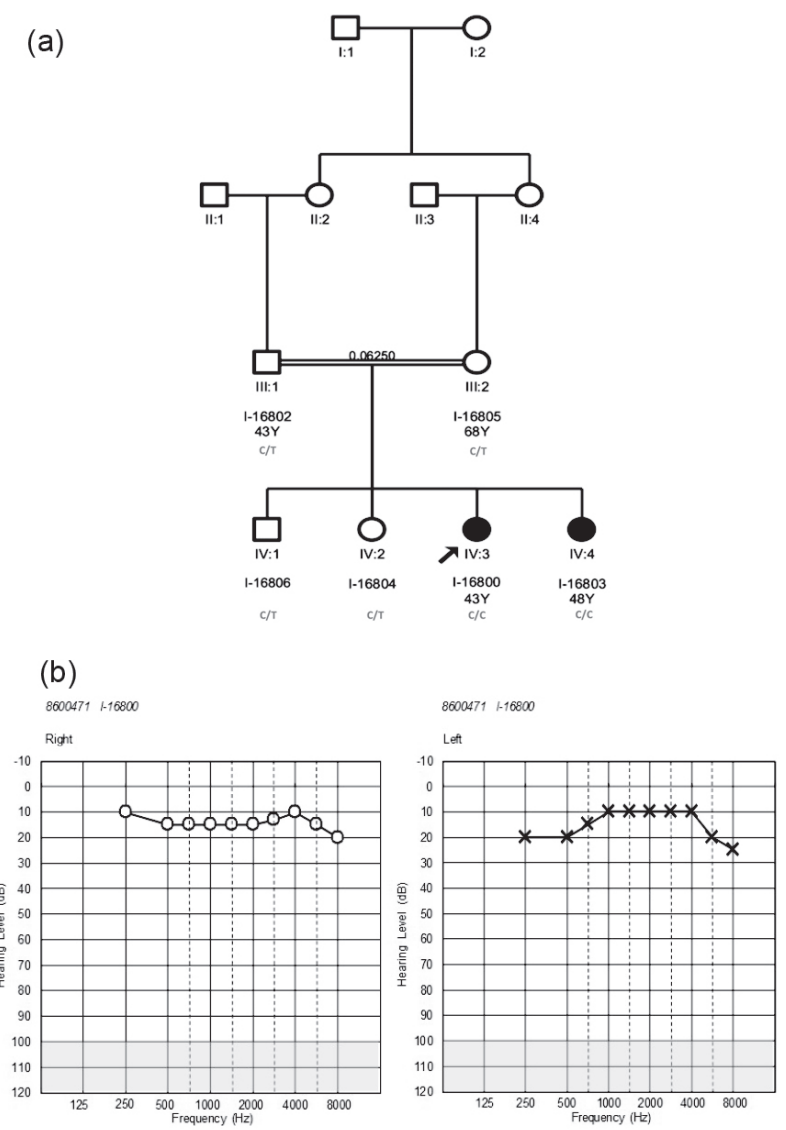

Figure 1. (a) Pedigree of family L-8600471. (b) Audiogram of proband IV:3 (I-16800). 
resulting in $72,46,20$, and 14 variants, respectively. Further investigation in 800 Iranian individuals (Iranome database) removed four variants with MAF $>0.01 \%$. The remaining variants were rechecked for quality with the Integrative Genomics Viewer (IGV) and two variants did not have sufficiently high quality. Out of the remaining eight variants, three were exonic, four intronic and one intergenic (Table 1). The three exonic variants included a synonymous variant in USP3 [MIM:604728] gene, a 3-bp variant in ZBED2 [MIM:615246] gene that was predicted to be a polymorphism, and a highly conserved and predicted deleterious missense variant in LSAMP [MIM:603241] gene (Table 2).

\section{Discussion}

MD is a complex disorder. It displays great inter- and intra-familial phenotypic heterogeneity and the exact pathomechanisms and genetic etiology underlying these phenotypes remain, for the most part, elusive. In this study, we utilized the power of exome sequencing on a

Table 1. Variants Identified in the Affected Individuals in Family L-8600471

\begin{tabular}{lll}
\hline Gene & Variant & Type \\
\hline OR5H2;OR5K4 & Chr3:98030901A $>C$ & Intergenic \\
ZBED2 & Chr3:111313042TCA $>-$ & Exonic (in frame deletion) \\
LSAMP & Chr3:115561402T >C & Exonic (nonsynonymous) \\
CFAP100 & Chr3:126152103C >- & Intronic \\
KMT2C & Chr7:152107907C >T & Intronic \\
USP3 & Chr15:63821105T >C & Intronic \\
USP3 & Chr15:63848878A $>C$ & Exonic (synonymous) \\
BAGE & Chr21:11088848A $>T$ & Intronic \\
\hline
\end{tabular}

Table 2. Predicted Deleterious Missense Variant in LSAMP [MIM:603241] Gene

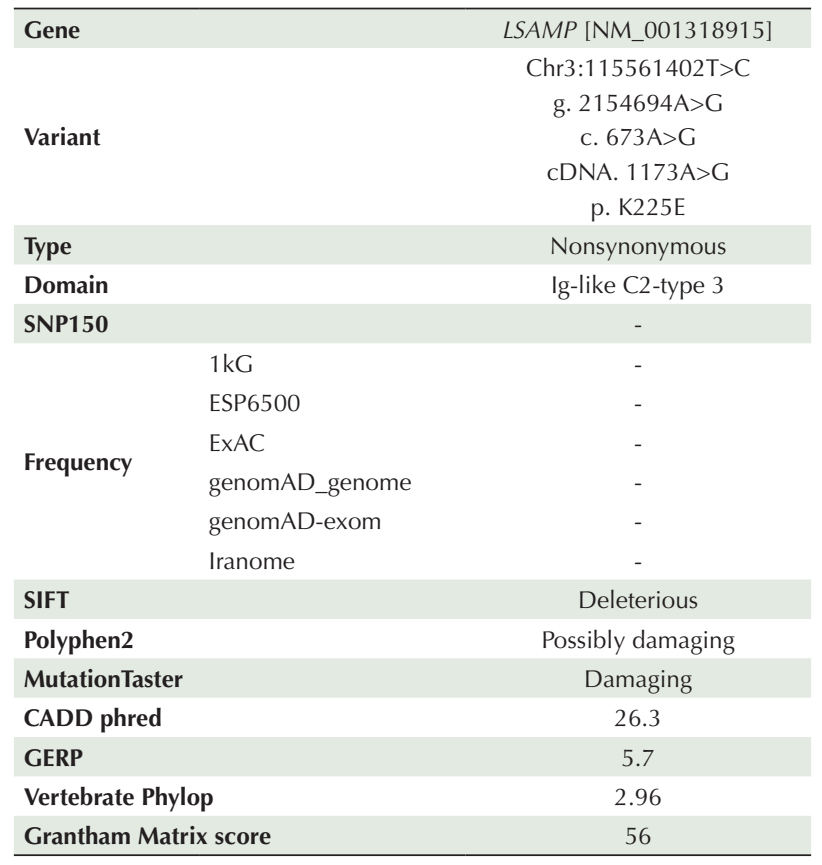

consanguineous Iranian family with apparent ARFMD to implicate the $L S A M P$ gene in the pathobiology of MD.

For decades, families from consanguineous regions have been used to investigate the molecular genetics of diseases. ${ }^{25-27}$ To this list of diseases, we add MD. We have identified a nonsynonymous variant (chr3:115561402T>C; c.673A>G; p.K225E) in the gene encoding the limbic system associated membrane protein (LSAMP) (Figure 2). Affected individuals were homozygous, and unaffected siblings and parents were heterozygous for this mutation (Figure 3). The p.K225E variant occurs in the highly conserved lysine 225 residue (Figure 4). The change from positively charged lysine to the negatively charged glutamic acid is predicted to be deleterious (Table 2) and most likely has a negative impact on local protein folding. It is predicted that this variant perturbs wild-type function either by disrupting a site for protein interactions or by altering folding.

LSAMP is a neuronal surface adhesion glycoprotein in cortical and subcortical regions of the limbic system. It takes part in neurite outgrowth, development of neuronal connection patterns and synaptic plasticity with an essential role in regulating the formation of septohippocampal, intrahippocampal, and thalamic connections. ${ }^{28-31} L S A M P$ is expressed in the hippocampus, cerebellum, eye, urogenital system, liver, heart, skeletal muscle, and small intestine. ${ }^{31-33}$ LSAMP is a candidate in the susceptibility to familial clustering of schizophrenia, bipolar disorder, and major depressive disorder. ${ }^{34}$ SNPs in LSAMP have been reported to have an association of genome-wide significance with ulcerative colitis in African Americans. ${ }^{33}$ LSAMP is also suggested to be a tumor suppressor and its genomic deletion is reported in aggressive prostate cancer, osteosarcoma, clear cell renal cell carcinoma, epithelial ovarian cancer, and acute myeloid leukemia. ${ }^{33,35,36}$ LSAMP downregulation has been shown to be associated with coronary artery disease. ${ }^{37}$ Animal studies have shown alteration in the regulation of emotional and social behavior in a changing environment in LSAMP-deficient mice. . $^{32,36,38}$

Cerebellum involvement has been established in cognitive and sensory motor processes such as maintaining balance, olfactory and auditory activities. ${ }^{39-42}$ It has been shown that PFL and vermis regions in the cerebellum are connected to auditory centers, response to sound and involvement in tinnitus perception. ${ }^{42,43}$ The potential role of structures in the limbic system in auditory processing and tinnitus has been suggested in various studies. ${ }^{44-48}$ The hippocampus, one of the structures in the limbic system, has a bilateral connection with auditory cortices to provide and receive inputs. ${ }^{46} \mathrm{Chan}$ et al showed that the hippocampus coordinates brain-wide neural activities including auditory activity at slow oscillation. ${ }^{44}$ Uchida et al suggested an association between hearing loss and hippocampal atrophy. ${ }^{49}$ Seo et al reported a significant 

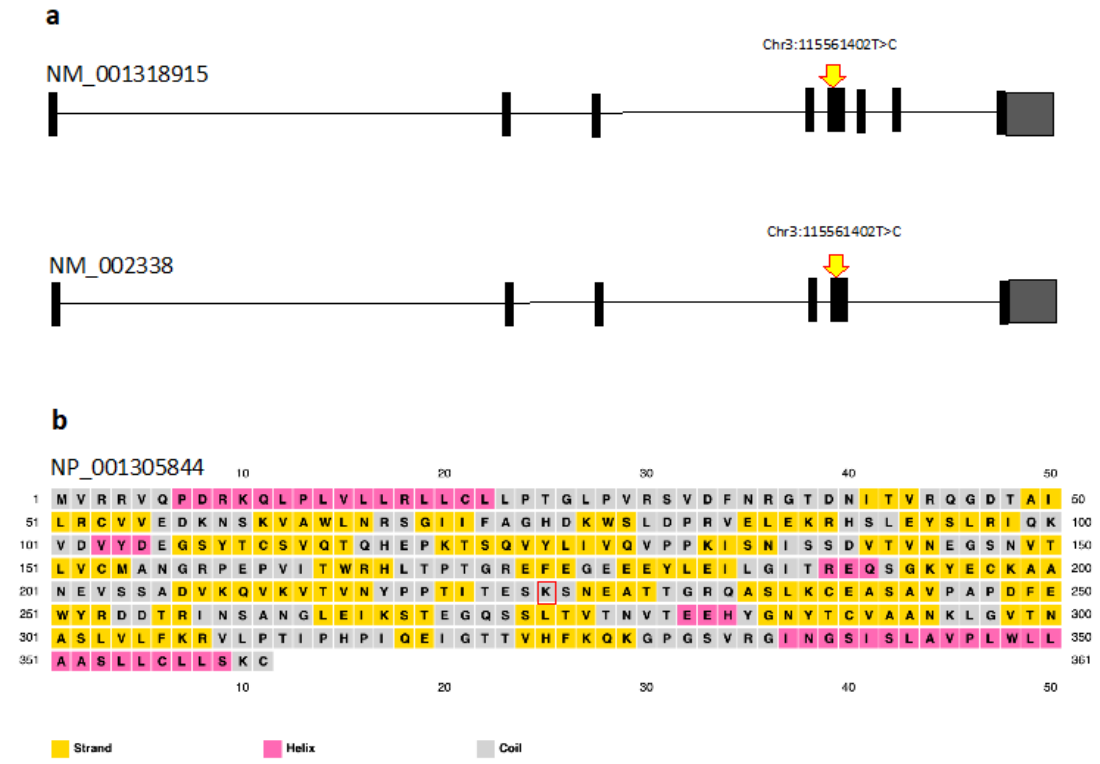

c

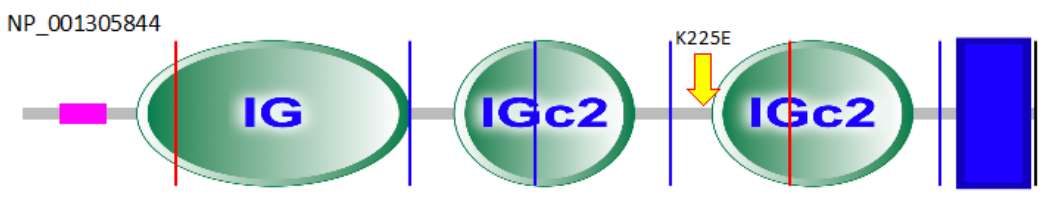

'o

100

'200

'300

Figure 2. (a) Two LSAMP transcripts adopted from UCSC genome browser. In each transcript, the location of the variant is shown by an arrow. (b) PSIPRED prediction for secondary structure of LSAMP protein. The lysine 225 residue is shown by a red rectangle. (c) SMART (a Simple Modular Architecture Research Tool) prediction for LSAMP domains. The arrow shows the location of the variant. Vertical lines show the location of introns.

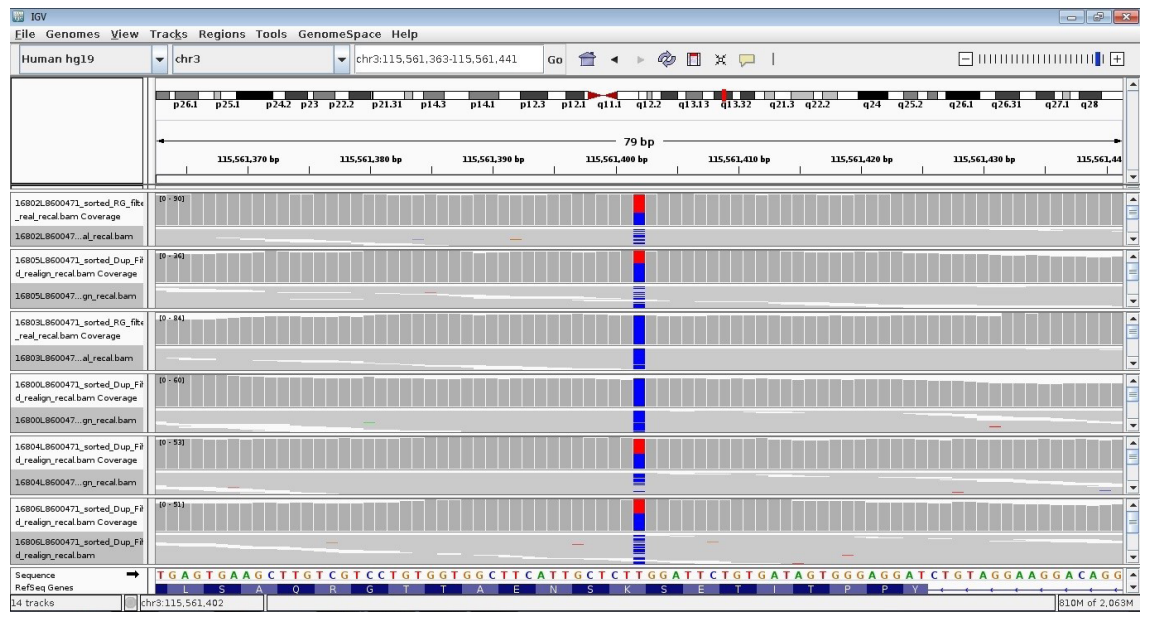

Figure 3. Integrative Genomics Viewer (IGV) image for the Chr3:115561402T>C variant in LSAMP gene in family L-8600471. From top to bottom, sequence results for two parents, two affected siblings and two healthy siblings are illustrated.

reduction in hippocampus volume in $\mathrm{MD}$ patients. ${ }^{50}$

Tinnitus is a network problem that involves the auditory and limbic regions of the brain including the amygdala, hippocampus, and parahippocampus. ${ }^{45-47,51,52}$ Chen et al showed that pharmacologically induced tinnitus implies increased connections between the auditory cortex, the cerebellum and the hippocampal gyrus. ${ }^{43}$ Zhang et al suggested that cholinergic signaling in the hippocampus, which modifies its synaptic plasticity or excitability, may be involved in the pathophysiology of tinnitus. ${ }^{46}$ Crippa et al observed different strengths of connections between the auditory cortex and limbic structures in patients with tinnitus. ${ }^{48}$ It has been shown that methylamine intoxication or bilateral resection of the hippocampus 


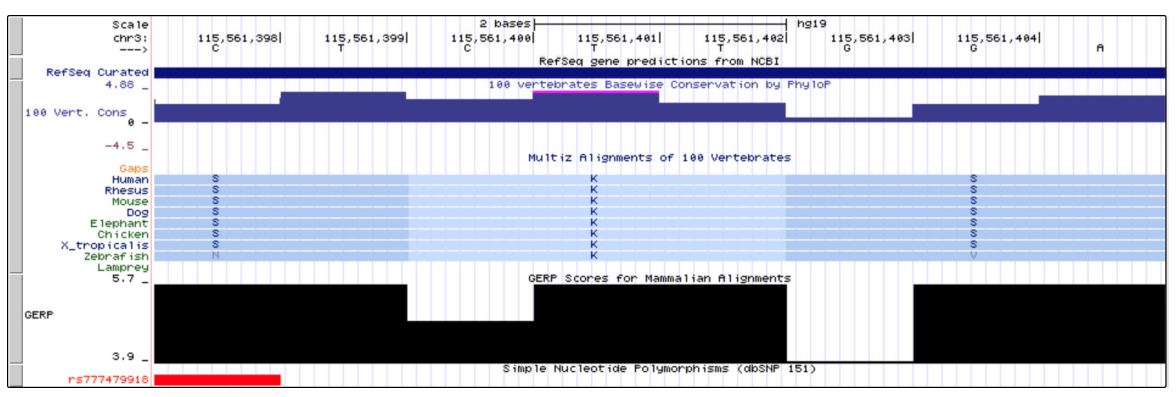

Figure 4. UCSC Genome Browser Entry for Human GRCh37 (hg19) chr3:115561402T Alongside The Flanking Regions and Amino Acid Products in Different Species.

result in tinnitus development. ${ }^{53,54}$

In summary, we have identified a promising candidate gene $(L S A M P)$ for ARFMD segregating in an Iranian family. The phenotype observed in the proband described here, i.e. vertigo, poor sense of smell, tinnitus, and borderline hearing ability, may originate from aberrant changes in the cerebellum and limbic system due to a deleterious mutation in the $L S A M P$ gene. Further studies, including screening of MD patients for variants in the LSAMP gene and functional studies of the pathomechanism driving the disease, are needed to fully clarify the role of LSAMP in MD.

\section{Authors' Contribution}

ZM: data analysis, writing and editing the original draft. KK: clinical assessment of patients, clinical validation of data, editing the original draft. Ma.M: whole exome sequencing. MA: whole exome sequencing. SA: sample recruitment, data profiling, clinical data recruitment of the samples, genetic consulting. KJ: DNA extraction, sample preparation. HN: Study design, editing the original draft, supervision. MF: clinical examination. Mo.M: clinical examination. AA: clinical examination. SM: clinical examination. AD: clinical assessment of patients, sample recruitment, editing of the original draft, supervision.

\section{Conflict of Interest Disclosures}

None declared.

\section{Ethical Statement}

This study was approved by the human research institutional review boards at the University of Social Welfare and Rehabilitation Sciences and Iran University of Medical Sciences, Tehran, Iran.

\section{Acknowledgements}

This study was funded by Iran National Science Foundation (INSF), grant no: 950022 and 92035782, Iran's National Elites Foundation (INEF), Deputy of Research, University of Social Welfare and Rehabilitation Sciences, Tehran, Iran, grant no. 95/801/T/3205, and Iran University of Medical Sciences, Tehran, Iran, grant no 3702. The authors would like to thank all family members and affected individuals who participated in this study.

\section{References}

1. Frejo L, Gallego-Martinez A, Requena T, Martin-Sanz E, AmorDorado JC, Soto-Varela A, et al. Proinflammatory cytokines and response to molds in mononuclear cells of patients with Meniere disease. Sci Rep. 2018;8(1):5974. doi: 10.1038/ s41598-018-23911-4.

2. Martin-Sierra C, Gallego-Martinez A, Requena T, Frejo L, Batuecas-Caletrio A, Lopez-Escamez JA. Variable expressivity and genetic heterogeneity involving DPT and SEMA3D genes in autosomal dominant familial Meniere's disease. Eur J Hum Genet. 2017;25(2):200-207. doi: 10.1038/ejhg.2016.154.

3. Vrabec JT. Genetic investigations of Meniere's disease. Otolaryngol Clin North Am. 2010;43(5):1121-32. doi: 10.1016/j.otc.2010.05.010.

4. Requena T, Espinosa-Sanchez JM, Cabrera S, Trinidad G, Soto-Varela A, Santos-Perez S, et al. Familial clustering and genetic heterogeneity in Meniere's disease. Clin Genet. 2014;85(3):245-52. doi: 10.1111/cge.12150

5. Committee on Hearing and Equilibrium guidelines for the diagnosis and evaluation of therapy in Menière's disease. American Academy of Otolaryngology-Head and Neck Foundation, Inc. Otolaryngol Head Neck Surg. 1995;113(3):181-5. doi: 10.1016/S0194-5998(95)70102-8

6. Frejo L, Giegling I, Teggi R, Lopez-Escamez JA, Rujescu D. Genetics of vestibular disorders: pathophysiological insights. J Neurol. 2016;263 Suppl 1:S45-53. doi: 10.1007/s00415-0157988-9.

7. Lopez-Escamez JA, Carey J, Chung WH, Goebel JA, Magnusson M, Mandala M, et al. Diagnostic criteria for Meniere's disease. JVestib Res. 2015;25(1):1-7. doi: 10.3233/VES-150549.

8. Chiarella G, Petrolo C, Cassandro E. The genetics of Meniere's disease. Appl Clin Genet. 2015;8:9-17. doi: 10.2147/TACG. S59024.

9. Greco A, Gallo A, Fusconi M, Marinelli C, Macri GF, de Vincentiis M. Meniere's disease might be an autoimmune condition? Autoimmun Rev. 2012;11(10):731-8. doi: 10.1016/j.autrev.2012.01.004.

10. Kim SH, Kim JY, Lee HJ, Gi M, Kim BG, Choi JY. Autoimmunity as a candidate for the etiopathogenesis of Meniere's disease: detection of autoimmune reactions and diagnostic biomarker candidate. PLoS One. 2014;9(10):e111039. doi: 10.1371/ journal.pone.0111039.

11. Hietikko E, Kotimaki J, Sorri M, Mannikko M. High incidence of Meniere-like symptoms in relatives of Meniere patients in the areas of Oulu University Hospital and Kainuu Central Hospital in Finland. Eur J Med Genet. 2013;56(6):279-85. doi: 10.1016/j.ejmg.2013.03.010.

12. Morrison AW, Bailey ME, Morrison GA. Familial Meniere's disease: clinical and genetic aspects. J Laryngol Otol. 2009;123(1):29-37. doi: 10.1017/S0022215108002788.

13. Arweiler-Harbeck D, Horsthemke B, Jahnke K, Hennies HC. Genetic aspects of familial Meniere's disease. Otol Neurotol. 2011;32(4):695-700. doi: 10.1097/MAO.0b013e318216074a.

14. Requena T, Cabrera S, Martin-Sierra C, Price SD, Lysakowski A, Lopez-Escamez JA. Identification of two novel mutations in FAM136A and DTNA genes in autosomal-dominant familial Meniere's disease. Hum Mol Genet. 2015;24(4):1119-26. doi: 10.1093/hmg/ddu524.

15. Kim BJ, Kim AR, Han KH, Rah YC, Hyun J, Ra BS, et al. Distinct vestibular phenotypes in DFNA9 families with $\mathrm{COCH}$ variants. Eur Arch Otorhinolaryngol. 2016;273(10):2993- 
3002. doi: 10.1007/s00405-015-3885-1.

16. Martin-Sierra C, Requena T, Frejo L, Price SD, GallegoMartinez A, Batuecas-Caletrio A, et al. A novel missense variant in PRKCB segregates low-frequency hearing loss in an autosomal dominant family with Meniere's disease. Hum Mol Genet. 2016;25(16):3407-3415. doi: 10.1093/hmg/ddw183.

17. Miller SA, Dykes DD, Polesky HF. A simple salting out procedure for extracting DNA from human nucleated cells. Nucleic Acids Res. 1988;16(3):1215. doi: 10.1093/ nar/16.3.1215.

18. Li H, Durbin R. Fast and accurate long-read alignment with Burrows-Wheeler transform. Bioinformatics. 2010;26(5):58995. doi: 10.1093/bioinformatics/btp698.

19. McKenna A, Hanna M, Banks E, Sivachenko A, Cibulskis K, Kernytsky A, et al. The Genome Analysis Toolkit: a MapReduce framework for analyzing next-generation DNA sequencing data. Genome Res. 2010;20(9):1297-303. doi: 10.1101/ gr.107524.110.

20. Van der Auwera GA, Carneiro MO, Hartl C, Poplin R, Del Angel $\mathrm{G}$, Levy-Moonshine A, et al. From FastQ data to high confidence variant calls: the Genome Analysis Toolkit best practices pipeline. Curr Protoc Bioinformatics. 2013;43:11.10.111.10.33. doi: 10.1002/0471250953.bi1110s43.

21. Wang $\mathrm{K}, \mathrm{Li}$ M, Hakonarson H. ANNOVAR: functional annotation of genetic variants from high-throughput sequencing data. Nucleic Acids Res. 2010;38(16):e164. doi: 10.1093/nar/gkq603.

22. Auton A, Brooks LD, Durbin RM, Garrison EP, Kang HM, Korbel JO, et al. A global reference for human genetic variation. Nature. 2015;526(7571):68-74. doi: 10.1038/nature15393.

23. Lek M, Karczewski KJ, Minikel EV, Samocha KE, Banks E, Fennell T, et al. Analysis of protein-coding genetic variation in 60,706 humans. Nature. 2016;536(7616):285-91. doi: 10.1038/nature19057.

24. SeattleWA. ExomeVariant Server, NHLBI GO Exome Sequencing Project (ESP). Available from: http://evs.gs.washington.edu/ EVS/. Accessed November 2017.

25. Kahrizi $\mathrm{K}, \mathrm{Hu} \mathrm{H}$, Hosseini $M$, Kalscheuer VM, Fattahi Z, Beheshtian M. Effect of inbreeding on intellectual disability revisited by trio sequencing. Clin Genet. 2019;95(1):151-159. doi: $10.1111 /$ cge.13463.

26. Sloan-Heggen $C M$, Babanejad $M$, Beheshtian $M$, Simpson AC, Booth KT, Ardalani F, et al. Characterising the spectrum of autosomal recessive hereditary hearing loss in Iran. J Med Genet. 2015;52(12):823-9. doi: 10.1136/ jmedgenet-2015-103389.

27. Najmabadi H, Hu H, Garshasbi M, Zemoitel T, Abedini SS, Chen W, et al. Deep sequencing reveals 50 novel genes for recessive cognitive disorders. Nature. 2011;478(7367):57-63. doi: 10.1038/nature10423.

28. Pimenta AF, Fischer I, Levitt P. CDNA cloning and structural analysis of the human limbic-system-associated membrane protein (LAMP). Gene. 1996;170(2):189-95. Doi: 10.1016/0378-1119(96)84698-1

29. Mann F, Zhukareva V, Pimenta A, Levitt P, Bolz J. Membraneassociated molecules guide limbic and nonlimbic thalamocortical projections. J Neurosci. 1998;18(22):940919. Doi:

30. Innos J, Philips MA, Raud S, Lillevali K, Koks S, Vasar E. Deletion of the Lsamp gene lowers sensitivity to stressful environmental manipulations in mice. Behav Brain Res. 2012;228(1):74-81. doi: 10.1016/j.bbr.2011.11.033.

31. Philips MA, Lillevali K, Heinla I, Luuk $\mathrm{H}$, Hundahl CA, Kongi $\mathrm{K}$, et al. Lsamp is implicated in the regulation of emotional and social behavior by use of alternative promoters in the brain. Brain Struct Funct. 2015;220(3):1381-93. doi: 10.1007/ s00429-014-0732-x.

32. Innos J, Philips MA, Leidmaa E, Heinla I, Raud S, Reemann P, et al. Lower anxiety and a decrease in agonistic behaviour in Lsamp-deficient mice. Behav Brain Res. 2011;217(1):21-31. doi: 10.1016/j.bbr.2010.09.019.

33. Brant SR, Okou DT, Simpson CL, Cutler DJ, Haritunians T, Bradfield JP, et al. Genome-wide association study identifies african-specific susceptibility loci in African Americans with inflammatory bowel disease. Gastroenterology. 2017;152(1):206-217.e2. doi: 10.1053/j.gastro.2016.09.032.

34. Behan AT, Byrne C, Dunn MJ, Cagney G, Cotter DR. Proteomic analysis of membrane microdomain-associated proteins in the dorsolateral prefrontal cortex in schizophrenia and bipolar disorder reveals alterations in LAMP, STXBP1 and BASP1 protein expression. Mole Psychiatry. 2009;14(6):601-13.

35. Kuhn MW, Radtke I, Bullinger L, Goorha S, Cheng J, Edelmann $\mathrm{J}$, et al. High-resolution genomic profiling of adult and pediatric core-binding factor acute myeloid leukemia reveals new recurrent genomic alterations. Blood. 2012;119(10):e6775 .

36. Baroy T, Kresse SH, Skarn M, Stabell M, Castro R, Lauvrak $\mathrm{S}$, et al. Reexpression of LSAMP inhibits tumor growth in a preclinical osteosarcoma model. Mol Cancer. 2014;13:93. doi: 10.1186/1476-4598-13-93.

37. Wang L, Hauser ER, Shah SH, Seo D, Sivashanmugam P, Exum ST, et al. Polymorphisms of the tumor suppressor gene LSAMP are associated with left main coronary artery disease. Ann Hum Genet. 2008;72(Pt 4):443-53. doi: 10.1111/j.14691809.2008.00433.x.

38. Innos J, Leidmaa E, Philips MA, Sutt S, Alttoa A, Harro J, et al. Lsamp(-)/(-) mice display lower sensitivity to amphetamine and have elevated 5-HT turnover. Biochem Biophys Res Commun. 2013;430(1):413-8. doi: 10.1016/j.bbrc.2012.11.077.

39. Grasby PM, Frith CD, Friston KJ, Bench C, Frackowiak RS, Dolan RJ. Functional mapping of brain areas implicated in auditory--verbal memory function. Brain. 1993;116(Pt 1):120.

40. Sens PM, Almeida Cl, Souza MM, Goncalves JB, Carmo LC. The role of the cerebellum in auditory processing using the SSI test. Braz J Otorhinolaryngol. 2011;77(5):584-8.

41. McLachlan NM, Wilson SJ. The Contribution of Brainstem and Cerebellar Pathways to Auditory Recognition. Front Psychol. 2017;8:265. doi: 10.3389/fpsyg.2017.00265. eCollection 2017.

42. Petacchi A, Laird AR, Fox PT, Bower JM. Cerebellum and auditory function: an ALE meta-analysis of functional neuroimaging studies. Hum Brain Mapp. 2005;25(1):118-28.

43. Chen YC, Li X, Liu L, Wang J, Lu CQ, Yang M, et al. Tinnitus and hyperacusis involve hyperactivity and enhanced connectivity in auditory-limbic-arousal-cerebellar network. Elife. 2015;4:e06576. doi: 10.7554/eLife.06576.

44. Chan RW, Leong ATL, Ho LC, Gao PP, Wong EC, Dong CM, et al. Low-frequency hippocampal-cortical activity drives brain-wide resting-state functional MRI connectivity. Proc Natl Acad Sci U S A. 2017;114(33):E6972-81. doi: 10.1073/ pnas.1703309114.

45. Gunbey HP, Gunbey E, Aslan K, Bulut T, Unal A, Incesu L. Limbic-Auditory Interactions of Tinnitus: An Evaluation Using Diffusion Tensor Imaging. Clin Neuroradiol. 2017;27(2):221230. doi: 10.1007/s00062-015-0473-0.

46. Zhang L, Wu C, Martel DT, West M, Sutton MA, Shore SE. Remodeling of cholinergic input to the hippocampus after noise exposure and tinnitus induction in Guinea pigs. Hippocampus. 2019;29(8):669-682. doi: 10.1002/ hipo. 23058.

47. Hinkley LB, Mizuiri D, Hong O, Nagarajan SS, Cheung SW. Increased striatal functional connectivity with auditory cortex in tinnitus. Front Hum Neurosci. 2015;9:568. doi: 10.3389/ fnhum.2015.00568.

48. Crippa A, Lanting CP, van Dijk P, Roerdink JB. A diffusion 
tensor imaging study on the auditory system and tinnitus. Open Neuroimag J. 2010;4:16-25.

49. Uchida Y, Nishita Y, Kato T, Iwata K, Sugiura S, Suzuki H, et al. Smaller Hippocampal Volume and Degraded Peripheral Hearing Among Japanese Community Dwellers. Front Aging Neurosci. 2018;10:319. doi: 10.3389/fnagi.2018.00319.

50. Seo YJ, Kim J, Kim SH. The change of hippocampal volume and its relevance with inner ear function in Meniere's disease patients. Auris Nasus Larynx. 2016;43(6):620-5. doi: 10.1016/j.anl.2016.01.006.

51. Salvi R, Langguth B, Kraus S, Landgrebe M, Allman B, Ding D, et al. Tinnitus and hearing loss and changes in hippocampus.
Seminars in Hearing. 2011;32(2):203-11

52. Vanneste S, Faber M, Langguth B, De Ridder D. The neural correlates of cognitive dysfunction in phantom sounds. Brain Res. 2016;1642:170-179. doi: 10.1016/j. brainres.2016.03.016.

53. Kreyberg S, Torvik A, Bjorneboe A, Wiik-Larsen W, Jacobsen D. Trimethyltin poisoning: report of a case with postmortem examination. Clin Neuropathol. 1992;11(5):256-9.

54. Corkin S, Amaral DG, Gonzalez RG, Johnson KA, Hyman BT. H. M.'s medial temporal lobe lesion: findings from magnetic resonance imaging. J Neurosci. 1997;17(10):3964-79. 